\title{
Compression deformation behaviors of sheet metals at various clearances and side forces
}

\author{
Mei Zhan ${ }^{1, a}$, Xianxian Wang ${ }^{1}$, Jian $\mathrm{Cao}^{2}$, and He Yang ${ }^{1}$ \\ ${ }^{1}$ College of Materials Science and Engineering, Northwestern Polytechnical University, \\ 127 Youyi Xilu, Xi'an, Shaanxi 710072, China \\ ${ }^{2}$ Department of Mechanical Engineering, Northwestern University, 2145 Sheridan Road, Evanston, \\ IL 60208, USA
}

\begin{abstract}
Modeling sheet metal forming operations requires understanding of plastic behaviors of sheet metals along non-proportional strain paths. The plastic behavior under reversed uniaxial loading is of particular interest because of its simplicity of interpretation and its application to material elements drawn over a die radius and underwent repeated bending. However, the attainable strain is limited by failures, such as buckling and inplane deformation, dependent on clearances and side forces. In this study, a finite element (FE) model was established for the compression process of sheet specimens, to probe the deformation behavior. The results show that: With the decrease of the clearance from a very large value to a very small value, four defects modes, including plastic t-buckling, microbending, w-buckling, and in-plane compression deformation will occur. With the increase of the side force from a very small value to a very large value, plastic t-buckling, w-buckling, uniform deformation, and in-plane compression will occur. The difference in deformation behaviors under these two parameters indicates that the successful compression process without failures for sheet specimens only can be carried out under a reasonable side force.
\end{abstract}

\section{Introduction}

For simulating metal forming processes, many new constitutive relations have been developed to describe materials behavior along non-proportional strain paths [1-8]. In order to fit these new constitutive models, experimental methods are required to test materials in an accurate, reliable and reproducible manner. The uniaxial tension-compression testing is of particular interest because of its simplicity of interpretation and uniformity of deformation over the entire sampled volume. However, the attainable strain in compression testing is severely limited by failures, such as buckling and in-plane deformation. Thus, various side support devices were proposed to suppress the buckling [9-11]. In these side support devices, the symmetric wedge device is the one that can completely eliminate the unsupported area [12]. However, the gap is fixed and the side force is uncontrollable in this symmetric

\footnotetext{
${ }^{\text {a }}$ Corresponding author: zhanmei@nwpu.edu.cn
}

This is an Open Access article distributed under the terms of the Creative Commons Attribution License 4.0, which permits unrestricted use, distribution, and reproduction in any medium, provided the original work is properly cited. 


\section{MATEC Web of Conferences}

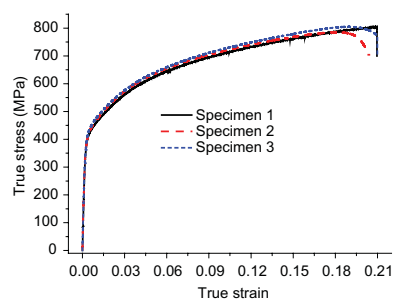

Figure 1. Uniaxial mechanical behaviors of DP600.

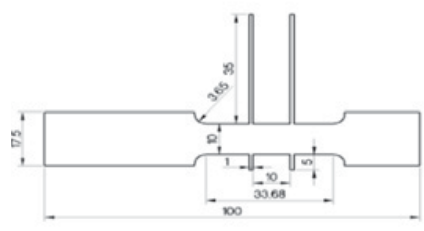

(a) Specimen with two pairs of fins

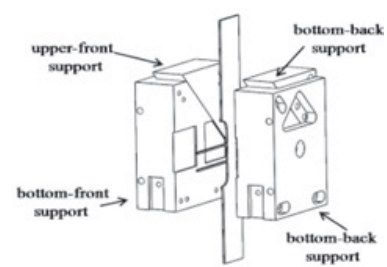

(b) Assembly of specimen and side force

Figure 2. Compression testing of sheet specimen.

wedge design. Therefore it is necessary to research the deformation behaviors of sheet metals under compression at various clearances and side forces for successful large-strain compression without failures.

In this study, a finite element (FE) model was established for the compression process of sheet specimens, to probe the deformation behavior. The influences of different initial clearances and side forces to sheet metal deformation were studied. Finally reasonable conditions are obtained to ensure the uniform deformation in sheet metal compression testing.

\section{Material and methods}

Dual Phase (DP 600) steel sheet metal was used in this study, which has a thickness of $1.0 \mathrm{~mm}$. Uniaxial tensile tests were conducted on normal specimens without fins using an MTS Sintech $20 \mathrm{~g}$ universal mechanical testing machine and a $25.4 \mathrm{~mm}$ MTS strain gauge. The elastic modulus of DP 600 is $182 \mathrm{GPa}$, and its plastic behavior obeying the Hollomon hardening law $\sigma=1085 \varepsilon^{0.1813}$ was determined from the uniaxial tensile tests by averaging from data in Fig. 1.

Since the implicit method is faster to obtain a steady-state and reliable solution than the explicit method is, the ABAQUS/standard software tool was used for finite element analyses for the compression test of the specimen with two pairs of asymmetry fins (Fig. 2) to probe the deformation behavior. Considering there are three directional deformations along the axial, width and thickness direction, three-dimensional (3D) analyses were performed. A $14 \mathrm{~mm}$ length region at each end of the specimen is clamped by a pair of clamps, and other regions of specimen are wrapped by four side supports (Fig. 2). The specimen, and four supports were modeled as separate parts. The specimen was defined as a $3 \mathrm{D}$ deformable solid body. The supports and clamps were defined as discrete rigid bodies. Each rigid body was assigned a reference point to represent its rigid motion in all degrees of freedom. The contact between the specimen and the supports was modeled with the CONTACT PAIR option, and the Coulomb friction formulation and penalty contact method were used. The supports were meshed by $3 \mathrm{D}$ bilinear rigid quadrilateral elements with four nodes. 3D linear reduction integration continuum 


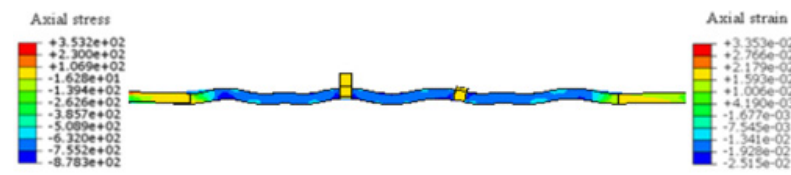

(a) Plastic t-buckling $\left(C_{\mathrm{i}}=0.5 \mathrm{~mm}\right)$.

(b) Local micro-bending $\left(C_{\mathrm{i}}=0.1 \mathrm{~mm}\right)$

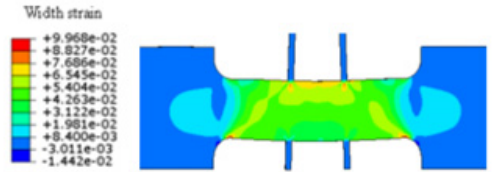

(c) w-buckling $\left(C_{\mathrm{i}}=0.1 \mathrm{~mm}\right)$

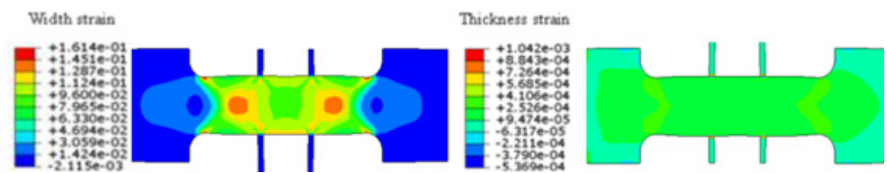

(d) In-plane deformation $\left(C_{\mathrm{i}}=0.0 \mathrm{~mm}\right)$.

Figure 3. Deformation behaviors under various clearances.

elements with eight nodes were used to discretize the specimen, and an enhanced hourglass control was employed to ensure computational precision. Initial geometric imperfections were imported to probe the buckling behaviors and other defects of sheet metals under compression. In the analyses, doubleprecision computations were carried out to improve simulation accuracy. To minimize the number of degrees of freedom and computation time, variable mesh densities were used for the specimen in this study. Using the meshing technology, the mesh size of $0.2 \mathrm{~mm}$ by $0.2 \mathrm{~mm}$ within the gauge region was used. Since this mesh density is close to that of Boger et al.'s study [13] and is much less than that of Cao et al.'s analysis (Cao et al. 2009, which is $0.75 \mathrm{~mm}$ by $0.75 \mathrm{~mm}$ ), the mesh technology used in this study can be used to capture the buckling, stress and strain distribution for the compression test of DP600 sheets.

Two parameters influencing the compression process are the initial clearance $\left(C_{i}\right)$ between the specimen and the supports, and the normal side force $\left(F_{n}\right)$ loading on the specimen. Deformation behaviors under various initial clearances $\left(C_{i}=0-0.5 \mathrm{~mm}\right)$ and normal side forces $\left(F_{n}=0.0-40 \mathrm{kN}\right)$ during $4 \mathrm{~mm}$ compression displacement (Dis) are analyzed.

\section{Results and discussion}

\subsection{Deformation behaviors under various clearances}

The simulations under various initial clearances $C_{i}$ show that the initial clearance has a significant influence on the deformation behaviors of the sheet specimen during compression. Under various initial clearances, four deformation modes, plastic t-buckling, micro-bending, w-buckling, and in-plane compression deformation (or barreling) will occur, as shown in Fig. 3. Under a given $C_{i}$ value, the occurrence of deformation mode and its transfer to others has been investigated as the compression displacement increasing.

When given an over-large clearance (such as $C_{i}=0.3-0.5 \mathrm{~mm}$ ), the compression in the beginning is similar to a free compression process. Therefore, with the increase of compression displacement, the axial compressive stress and strain increase. When the stress and strain reach their critical values, some mode plastic t-buckling (Fig. 3(a)) occurs with a very small increase in displacement according to compression bar buckling theory of Euler. Thus a decrease in the crosshead force and reaction forces on supports occurs (Fig. 4(a) and (b)). After the plastic t-buckling occurring, the unstable state and the decrease in forces will continue for some time till the specimen contacts with the supports, then the forces will increase resulting from supports until the next mode t-buckling occurs due to that the 


\section{MATEC Web of Conferences}

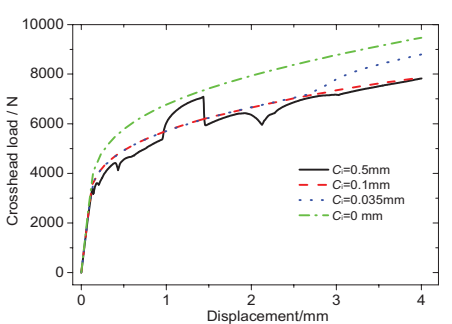

(a) Crosshead force

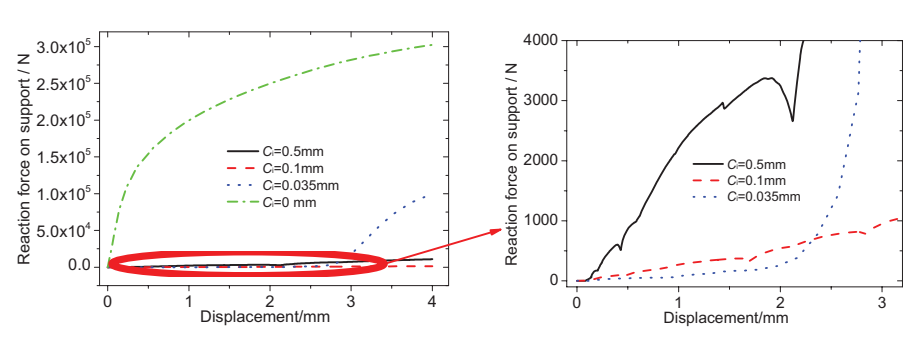

(b) Reaction force

Figure 4. Crosshead forces and reaction forces on supports verse displacement at various clearances.

constraint from the normal direction is not sufficient to support this mode plastic stable deformation out of plane from normal direction (Fig. 4(a) and (b)).

As the clearance decreases a little from the occurrence condition of plastic t-buckling but still a large value (such as $C_{i}=0.1 \mathrm{~mm}$ ), the decrease in clearance will suppress the obvious plastic t-buckling and thus the loading force can be transfer smoothly. However, local micro-bending occurs in the early and midst period of compression (Fig. 3(b)). This local micro-bending is similar to the plastic t-buckling with very low wave. As the compression going on, w-buckling occurs (Fig. 3(c)) according to compression bar buckling theory of Euler. During the whole process, there is almost no abnormal variation in the crosshead force (Fig. 4(a)) but the abnormal variation in the reaction force (Fig. 4(b)) of the supports is still obvious.

As the clearance decreases continually to a small value (such as $\mathrm{Ci}=0.035 \mathrm{~mm}$ ), local microbending still occurs in the early and midst period due to that the small clearance in this period, and in-plane compression occurs in the last period due to the increase in thickness in this period make the real clearance becomes less and less, as shown in Fig. 3(d). This leads to negligible abnormal variation in the loading force (Fig. 4(a)) but still noticeable variation in the reaction force on the supports in the early and midst period, and a dramatic increase in these forces in the last period (Fig. 4(b)).

As the clearance continually decreases to an over-small value (such as $\mathrm{C}=0 \mathrm{~mm}$ ), in-plane compression deformation occurs in the beginning of compression and becomes more and more obvious until barreling occurs (Fig. 3(d)) due to that the normal constraint from supports will constrain excessively the thickness deformation. In the case, there is no abnormal variation in the crosshead force and the reaction force on the supports in the whole period, as shown in Fig. 4(a) and (b). However, the values of these forces are larger a lot than those under other clearances (Fig. 4(a) and (b)).

\subsection{Deformation behaviors under various side forces}

The simulations under various side forces show that the side force also has a significant influence on the deformation behaviors of the sheet specimen during compression. With the increase of the side force, four deformation modes, including plastic t-buckling, w-buckling, uniform deformation, and in-plane compression deformation, will occur successively, as shown in Fig. 5.

The plastic t-buckling occurs when the normal side force $F_{n}$ is such a small value (for example, $F_{n}=0.03 \mathrm{kN}$ ), as shown in Fig. 5(a). Under this deformation behavior, the constraint from the normal direction is too small to support the plastic stable deformation out of plane. As the side force increases on the occurrence condition of plastic t-buckling (such as $F_{n}=1.25-3.75 \mathrm{kN}$ ), w-buckling will occur (Fig. 5(b)) due to that the increase in side force is enough to suppress the plastic t-buckling and thus the loading force can be transfer smoothly. With the increase in the side force, the thickness strain reduces and width strain increases, thus the ratio of width strain to thickness strain increases, as shown in Fig. 6. Figure 6 shows that when $F_{n}=5-10 \mathrm{kN}$, most of ratios are in the range of $0.95-1.1$, when 
(a) Plastic t-buckling under over-small side force $\left(F_{\mathrm{n}}=0.03 \mathrm{kN}\right)$.

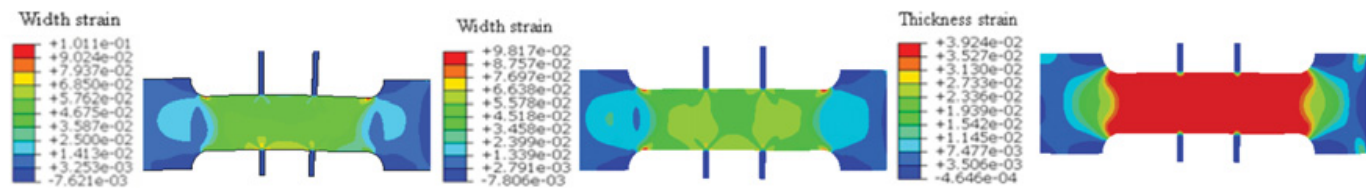

(b) w-buckling under small side force $\left(F_{\mathrm{n}}=1.25 \mathrm{kN}\right)$ (c) In-plane deformation under over-large side force $\left(F_{\mathrm{n}}=40.0 \mathrm{kN}\right)$.
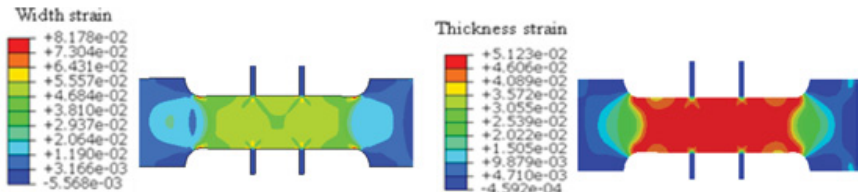

(d) Uniform deformation behavior under reasonable side force $\left(F_{\mathrm{n}}=5 \mathrm{kN}\right)$.

Figure 5. Deformation behavior under various side forces.

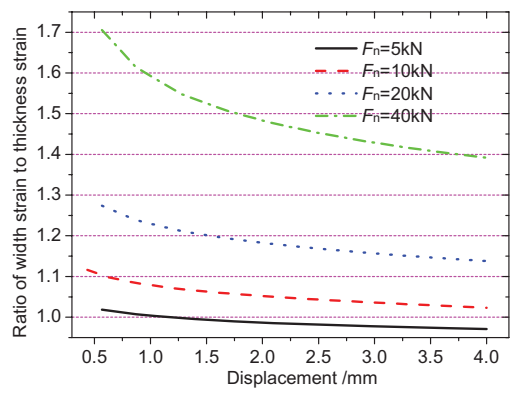

Figure 6. Ratios of width strain to thickness strain under various side forces.

$F_{n}=20 \mathrm{kN}$, most of ratios are in the range of $1.1-1.3$, and when $F_{n}=40 \mathrm{kN}$, most of ratios are in the range of 1.4-1.7. This means that as the side force increases excessively (such as $F_{n}=20-40 \mathrm{kN}$ ), in-plane compression deformation even barreling will occur due to excessive constraint in the thickness deformation, as shown in Fig. 5(c). Uniform deformation occurs when a reasonable side force (such as $F_{n}=5-20 \mathrm{kN}$ ) is applied, as shown in Fig. 5(d). In this case, the unstable deformation behaviors can be suppressed and the deformation along the thickness direction can't be constrained excessively, thus in-plane compression deformation also can be suppressed.

Figure 7 shows that the side force when in-plane compression deformation occurring is larger a lot than other side forces. When the plastic t-buckling occurs under a small side force there is only a decrease in the axial loading force. This is different from that when the plastic t-buckling occurs under an over-large clearance, where there is usually an increase followed by the decrease in the force (Fig. 4(a)). This is due to that once the plastic t-buckling occurs under a small side force, the unstable state will continue since the side force is insufficient to suppress the unstable state. 


\section{MATEC Web of Conferences}

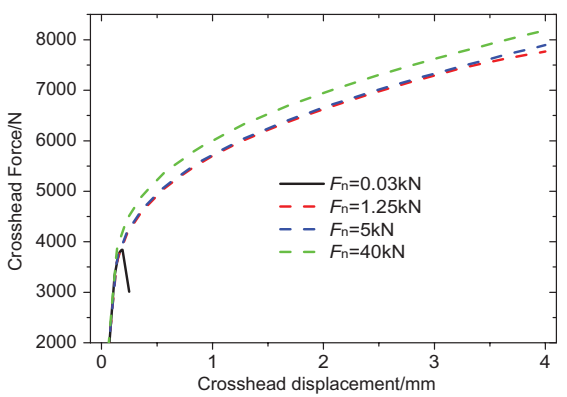

Figure 7. Crosshead loads verse displacement under various side forces.

(a)

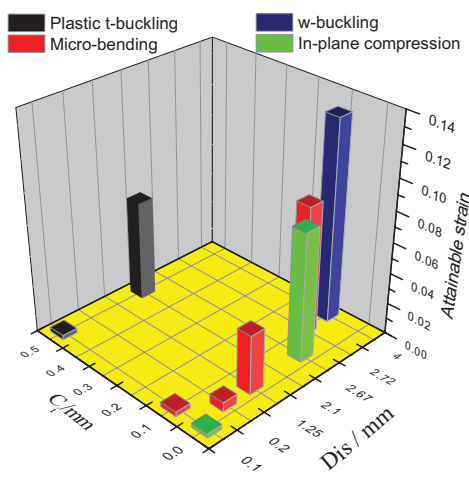

(b)

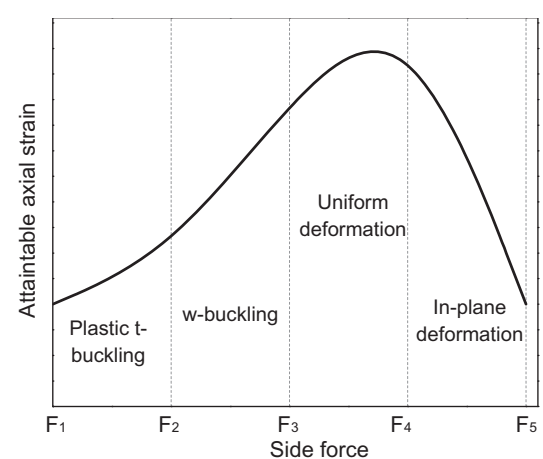

Figure 8. Schematic of relationship of deformation behaviors with (a) initial clearance and (b) normal side force.

\subsection{Relationship of deformation behaviors with initial clearance and side force}

Based on the above results, the relationship of deformation behaviors under various clearances and side forces can be schematized as Fig. 8. Under an over-large clearance, plastic t-buckling from low mode to high mode will occur as compression going on. Under a large clearance, micro-bending occurs in the early and midst period and w-buckling occur in the last period of compression. Under a small clearance, in-pane deformation occurs in the last period of compression. Under an over-small clearance, in-plane deformation will occur in the beginning and become more and more obvious with the process going on. Figure 8(b) shows plastic t-buckling, w-buckling, uniform deformation, and in-plane compression deformation that occur successively as the side force increasing.

Comparison in Fig. 8(a) and (b) shows that the difference of the two parameters is that uniform deformation behavior desired will only occur under a reasonable side force. This is due to that a reasonable side force can give the specimen a proper support to suppress the unstable deformation and make the supports move normally to let the specimen thicken "freely" which can avoid in-plane compression deformation. However, a given initial clearance is difficult to attain both of goals.

\section{Conclusion}

With the decrease of the clearance from a very large value to a very small value, four defects modes, including plastic t-buckling, micro-bending, w-buckling, and in-plane compression deformation will occur. While with the increase of the side force, plastic t-buckling, w-buckling, uniform deformation, and in-plane compression will occur. And uniform deformation occurs when there is no initial clearance 
and a reasonable normal side force is applied because of the unstable deformation behaviors suppressed and the deformation along the thickness direction not constrained excessively.

The research is funded by National Science Fund for Excellent Young Scholars of China (51222509), the National Natural Science Foundation of China (51175429), "973" National Basic Research Project of China (No. 2011CB012804). This work was also supported by China Scholarship Council.

\section{References}

[1] L. Geng, R.H. Wagoner, Int. J. Mesh, 44, 123 (2002)

[2] G. Kang, N. Ohno, A. Nebu, Int. J. Plast. 19, 1801 (2003)

[3] O.U. Colak, Int. J. Plast. 20, 1387 (2004)

[4] L. Sun, R.H. Wagoner, Int. J. Plast. 45, 174 (2013)

[5] F. Yoshida, H. Hamasaki, T. Uemori, Procedia Engineering 81, 1261 (2014)

[6] T. Uemori, S. Sumikawa, T. Naka, F. Yoshida, Procedia Engineering 81, 933 (2014)

[7] Y.H. Xiao, Z.H. Cui, H.B. Yin, C. Guo, Procedia Engineering 81, 1277 (2014)

[8] W. Ramberg, J.A. Miller, J. Aeronaut. 13, 569 (1946)

[9] L. Dietrich, K. Turiski, Rozprawy Inzynierskie 26, 91 (1978)

[10] F. Yoshida, T. Uemori, K. Fujiwara, Int. J. Plast. 18, 633 (2002)

[11] T. Kuwabara, Numisheet A, 20 (2005)

[12] J. Cao, W. Lee, H.S. Cheng, M. Seniw, H.P. Wang, K. chung, Int. J. Plast. 25, 942 (2005)

[13] R.K. Boger, R.H. Wagoner, F. Barlart, M.G. Lee, K. Chung, Int. J. Plast. 21, 2319 (2005) 